\title{
Ein diagnostisches Modell zur Berechnung von Trajektorien über dem Schweizer Mittelland
}

\begin{abstract}
Summary
The aim of the presented model (called WITRA) is to use the data of the automatic wind stations on the Swiss Plateau as a basis for calculating back-trajectories over a complex terrain. WITRA interpolates a three-dimensional windfield out of the measured data and the radio-sonde profile of Payerne. By a mathematical method developed by SASAKI (1958) based on the variational analysis and with regard to its application to the investigated problem by SHERMAN (1978), the windfield is slightly adjusted in a least-square sense in order to get it free of divergence, i. e. mass-consistent. Within the same step the complex terrain and its effects on the wind are taken into account. Trajectories can be calculated by a method mentioned by PETTERSSEN (1956) using a sequence of these windfields.
\end{abstract}

\section{Einleitung}

Das vorliegende Modell wurde in der Absicht entwickelt, ein relativ einfaches Instrument zur Verfügung zu stellen, um aus vorhandenen Routine-Meßdaten (automatisches meteorologisches Meßnetz der Schweiz ANETZ, Radiosondierung von Payerne) dreidimensionale, divergenzfreie Windfelder und mit deren Hilfe Partikel-Trajektorien berechnen zu können. Das Untersuchungsgebiet des Schweizer Mittellandes umfaßt ca. $90 \times 220 \mathrm{~km}^{2}$ und darin 20 ANETZ-Stationen, welche 10-Minuten-Mittelwerte liefern. Damit ist die zeitliche Auflösung überhaupt kein Problem, die räumliche Auflösung ist mit einer Station pro $1000 \mathrm{~km}^{2} \mathrm{zwar}$ so gut wie nirgendwo sonst (ausser während kurzfristigen Intensivmeßkampagnen), doch für eine komplexe Topographie wie das Schweizer Mittelland trotzdem nur knapp genügend. Die Vertikalmessungen stellen sowohl aus zeitlicher (nur vier Sondierungen pro Tag) als auch aus räumlicher Sicht (nur 1 Sondierung: Payerne) ein Problem dar. Diese Vorgaben bedeuten bereits eine erhebliche Einschränkung, was die Anwendbarkeit eines solchen Modells anbetrifft: Wegen der räumlichen Auflösung muß das Windfeld relativ homogen sein, und dies ist nur bei Windgeschwindigkeiten von mehr als 1,5-2 m/s der Fall. Zusätzlich müssen die Vertikalgeschwindigkeiten relativ klein sein, da konvektive Strömungen wegen fehlender Messungen nicht erfaßt werden können. Diese Bedingungen sind praktisch nur bei winterlichen Hochnebellagen erfüllt. Ebenfalls problematisch sind Angaben zur unteren Grenzschicht ( (Surface Layer»), da hier ebenfalls kleinräumige Prozesse von großer Bedeutung sind, welche sowohl unterhalb der Meßauflösung als auch unterhalb der Maschenweite des Modells liegen.

\section{Datengrundlagen und -bearbeitung}

Als Grundlage werden wie erwähnt die Daten der ANETZ-Stationen verwendet, welche jedoch gewisse Bedingungen erfüllen müssen: Die Windmessung muß für eine größere Region einigermaßen "repräsentativ». sein, d. h. es dürfen keine störenden Lokaleinflüsse vorhanden sein wie z. B. größere Gebäude oder Bäume in einem geringeren Abstand als dem 10fachen ihrer Höhe sowie größere topographische Hindernisse, welche das Windfeld am Meßstandort bedeutend beeinflussen. Aus diesen Gründen können die Stationen Luzern, Tänikon, Adelboden und Engelberg nicht berücksichtigt werden. Die Stationen Interlaken und Altdorf hingegen liegen in Tälern, welche eine ganze Region prägen, und liefern trotz auftretenden Kanalisierungseffekten brauchbare Daten. Die verwendeten Stationen sind in Figur 1 dargestellt. Ebenfalls berücksichtigt werden muß mit einem Korrekturfaktor die Geschwindigkeitserhöhung über einzelstehenden Gebäuden.

Als Grundlage für das Relief werden die je nach Gitternetz-Auflösung geglätteten Daten des RIMINI-Rasters verwendet.

Die Vertikalstruktur des (Horizontal-)Windfeldes wird aus den Radiosondierungen (mittag und mitternachts) und den Pilotballonflügen (6 Uhr und $18 \mathrm{Uhr}$ ) der meteorologischen Station in Payerne gewonnen.

Die meist auf 10 bis 15 m Höhe über Boden (bzw. über dem Dach eines Gebäudes) erhobenen Daten werden mit Hilfe des sogenannten «Power-Law-Profils» nach der Formel

$\mathrm{v}(\mathrm{z})=\mathrm{v}(\mathrm{h}) *(\mathrm{z} / \mathrm{h})^{\mathrm{p}}$

auf die Höhe der Obergrenze der Surface Layer extrapoliert, wobei v die Windgeschwindigkeit in der Höhe $\mathrm{z}$ (Schichtobergrenze) bzw. h (Meßhöhe) bezeichnet. Der Exponent $p$ ist abhängig von der Stabilität der Schichtung innerhalb der «Surface Layer» und kann empirisch

Neu Urs, Lic. phil. nat., Geograph, Geographisches Institut, Hallerstraße 12, CH-3012 Bern 


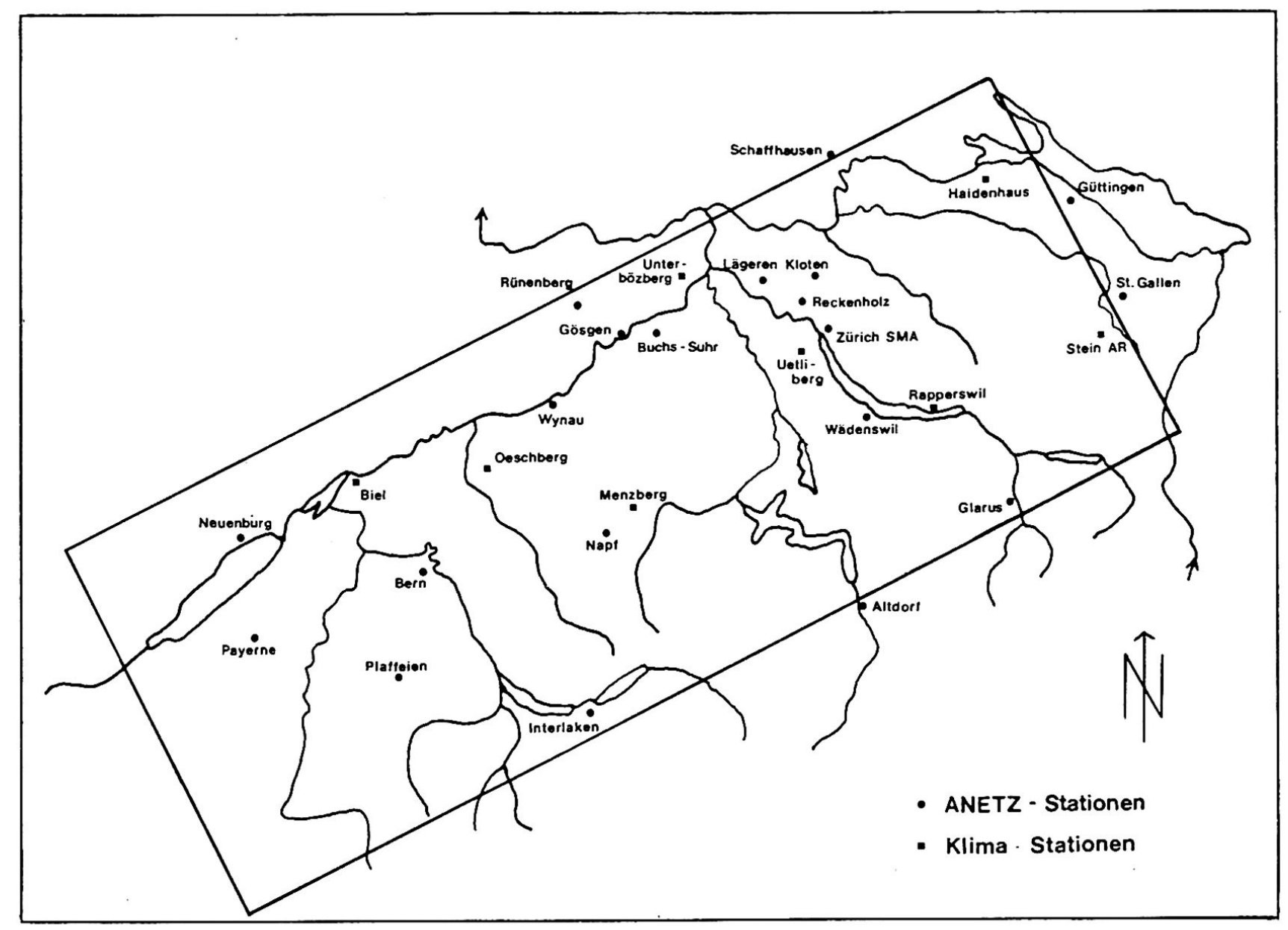

Fig.1 ANETZ-Stationen für die Berechnung des Windfeldes

bestimmt werden aus den Daten des 110 Meter hohen Meteo-Turmes des Kernkraftwerkes Gösgen. Da das Modell nur in einem beschränkten Bereich möglicher Wetterlagen arbeitet, ist die Schichtung meist sehr ähnlich ( $p$ liegt zwischen 0.1 und 0.13 für neutrale Schichtung).

\section{Modellbeschreibung}

Der grobe Aufbau des Modells ist in Fig. 2 ersichtlich. Verwendet wird ein karthesisches Koordinatensystem. Heute wird in ähnlichen Modellen häufig ein dem Terrain folgendes Koordinatensystem verwendet, doch bei einer am Seitenrand in die Topographie übergehenden Obergrenze des Modells ergeben sich damit große Schwierigkeiten.

\subsection{Modellbereich}

Der Modellbereich deckt mit einem Rechteck das schweizerische Mittelland mit Ausnahme des Genferseebeckens ab (siehe auch Fig. 2). Aus topographischen Gründen wurde die x-Achse parallel zur Achse des Mittellandes gelegt, also etwa in der Achse Westsüd-
Extrapolation der Messdaten von lom ü. Boden über die obergrenze der "Surface Layer" (ca. $100 \mathrm{~m} \mathrm{ü.B.}$

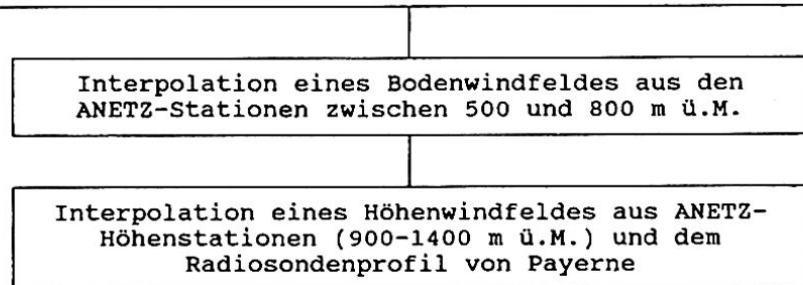

Vertikalinterpolation zwischen Boden- und Höhenwindfeld unter Berücksichtigung des profils von Payerne

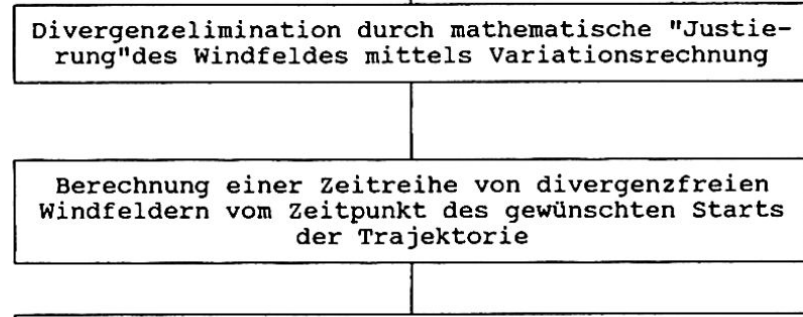

Berechnung der Trajektorie nach der "PetterssenMethode"

Fig. 2 Übersicht über die Einzelschritte von WITRA 
west-Ostnordost. Die Koordinaten der Eckpunkte lauten:

$560500 / 127000-757500 / 225500$

$523000 / 202000-720000 / 300500$

In der Vertikalrichtung wird der Bereich gegen unten durch die Topographie und gegen oben durch die erste markante Temperaturinversion, welche für jede Modellrechnung bestimmt werden muß, begrenzt. Die Horizontalauflösung beträgt $1,1 \mathrm{~km}$ oder höher (wählbar), die Vertikalauflösung $100 \mathrm{~m}$.

\subsection{Interpolation}

\section{a) Interpolation des Bodenwindfeldes}

Alle Stationen im Mittelland liegen zwischen 400 und $600 \mathrm{~m}$ ü. M., mit Ausnahme der Station St. Gallen, die an einem wenig steilen Hang auf 790 m ü. M. liegt, jedoch auch das Windfeld über der Stadt einigermaßen repräsentieren sollte. Aus diesen Messungen wird ein zweidimensionales, sog. "Bodenwindfeld» interpoliert. Das heißt, für jeden Gitterpunkt $(i, j)$ werden die Windkomponenten der drei nächstliegenden Stationen gemittelt, und zwar gewichtet mit $1 / \mathrm{d}^{3}$ (i bezeichnet die $\mathrm{x}-$, j die $y$-Koordinate, d den Abstand des betrachteten Gitterpunktes von der jeweiligen Station.)

Die Windrichtungskomponente in $x-$ Richtung, $U(i, j)$, wird berechnet aus

$U(i, j)=\sum U_{n} / d_{n}(i, j)^{3} / \sum 1 / d_{n}(i, j)^{3}$,

als Summe über die drei nächstliegenden Stationen, wobei $U_{n}$ die $x$-Komponente der Station $n$ und $d_{n}(i, j)$ die Distanz der Station n zum Gitterpunkt (i, j) bezeichnet. Die y-Komponente V $(\mathrm{i}, \mathrm{j})$ wird analog berechnet.

\section{b) Interpolation des Höhenwindfeldes}

Für das Höhenwindfeld stellt sich das Problem, daß einigermaßen brauchbare Windmessungen in größeren Höhen nur sehr spärlich vorhanden sind. Die Gipfelstationen sind sehr häufig durch thermisch bedingte Lokalwinde beeinflußt (RICKLI et al. 1989) und weisen zudem «mechanische» Störungen durch den unter ihnen liegenden Hügel auf, Stationen in hochgelegenen Tälern zeigen ein stark kanalisiertes Windfeld (Adelboden oder Engelberg beispielsweise).

Zudem liegen die meisten der in Frage kommenden Gipfelstationen (Moléson, Pilatus, Säntis) in Höhenbereichen, die für eine winterliche Grenzschicht nicht mehr in Betracht fallen. Die einzige verwendbare Höhenstation war lange Zeit der Napf (1400 m ü. M.), ein bewaldeter Hügel im Emmental. Seit einem Jahr stehen nun auch die Stationen Plaffeien-Oberschrot (ca. $1000 \mathrm{~m}$ ü. M.) und Lägeren (ca. $900 \mathrm{~m}$ ü. M.) in Betrieb. In absehbarer Zeit wird auch die Station des sog. Ergänzungsnetzes ENET auf dem Uetliberg den Betrieb aufnehmen. Als weiterer Meßpunkt werden die im entsprechenden Niveau der Payerne-Sondierung gemessenen bzw. zeitlich interpolierten Werte verwendet. Als dritter Meßpunkt für frühere Zeitpunkte werden Windgeschwindigkeit und -rich- tung oberhalb der Station KKW Gösgen extrapoliert, weil hier wenigstens eine Messung in bereits größerer Höhe (110 m über Grund) existiert und damit ein Unsicherheitsfaktor, nämlich die Extrapolation der Bodenwindmeßung an die Obergrenze der Surface Layer, wegfällt. Die Extrapolation auf Höhe des Napf erfolgt in Angleichung an das Payerne-Profil. Aus diesen Meßpunkten wird wiederum, nach der gleichen Methode wie beim Bodenwindfeld, ein horizontales Höhenwindfeld inter-bzw. extrapoliert.

\section{c) Vertikalinterpolation}

Die Obergrenze der Mischungsschicht wird über dem ganzen Mittelland als ungefähr aufder gleichen Höhe liegend $( \pm 100 \mathrm{~m})$ angenommen. Diese Annahme ist bei winterlichen Hochdruck- und Bisenlagen, d. h. bei eingeschränktem vertikalem Austausch, welche mit WITRA vor allem untersucht werden sollen, annähernd gegeben. Die Bestimmung der Obergrenze der Mischungsschicht ist mit einem Routineverfahren kaum möglich oder wäre jedenfalls sehr aufwendig, da viele Spezialfälle berücksichtigt werden müßten. Die Mischungsschichthöhe $\mathrm{MH}$ muß also für jeden Fall speziell bestimmt werden.

MH wird für die Zeitpunkte Mittag und Mitternacht aus dem Temperaturprofil der Payernesondierungen bestimmt. Für die Zeitpunkte zwischen den Messungen muß die Mischungsschichthöhe geschätzt werden. Dabei müssen u. a. die Wetterlage und der zeitliche Verlauf der Temperatur berüchsichtigt werden.

Die Interpolation zwischen Bodenwindfeld und Höhenwindfeld geschieht durch lineare Interpolation und anschließend gewichtete Angleichung an das Payerne-Profil unter Berücksichtigung der Entfernung von der Station Payerne. Das heißt, je weiter ein Punkt von Payerne weg liegt, um so schwächer wird das Profil angepaßt. In großer Entfernung sowie für Punkte, welche wegen topographischer Hindernisse keine "Sichtverbindung» mit Payerne haben, erfolgt die Interpolation linear.

\section{d) Einbezug der Topographie}

Die bisher berechneten Windfelder weisen für jeden Gitterpunkt einen Windvektor auf, auch für diejenigen Punkte, welche unterhalb der Bodenoberfläche liegen. Die Topographie wird nun in das Windfeld einbezogen, indem für alle Gitterpunkte, welche sich an oder unterhalb der Bodenoberfläche befinden, den Windkomponenten der Wert 0 zugeordnet wird. Da sich alle Bodenstationen auf ungefähr derselben Höhe befinden (zwischen 400 und $600 \mathrm{~m}$ ü. M.), werden die Bodenstationen alle dem Niveau $\mathrm{k}=3$ (600 $\mathrm{m}$ ü. M.) zugeordnet. Das Niveau $k=1$ ( $400 \mathrm{~m}$ ü. M.) weist für alle Punkte den Windwert 0 auf, da kein Punkt im Mittelland eine tiefere Geländehöhe aufweist. Den relativ wenigen Punkten des Niveaus $k=2$, welche einen Windwert aufweisen, wird derselbe Wert zugeordnet wie im Niveau $k=3$.

\section{e) Vertikalgeschwindigkeiten}

Mangels Ausgangsdaten werden die Vertikalgeschwindigkeiten für alle Gitterpunkte des interpolierten Initialwindfeldes gleich 0 gesetzt. 
Die Vertikalgeschwindigkeiten im berechneten Windfeld beruhen also allein auf der Bedingung der Divergenzfreiheit und werden durch die entsprechenden Korrekturen erzeugt.

\subsection{Divergenzelimination}

Die Divergenzelimination erfolgt nach dem Prinzip der Variationsrechnung. Im folgenden werden die dazu verwendeten theoretischen Grundlagen, wie sie von SASAKI (1958) und SHERMAN (1978) beschrieben werden, kurz erläutert:

Bei der Konstruktion von meteorologischen Feldern, seien dies nun Wind-, Temperatur- oder Stoffkonzentrationsfelder, ist das primäre Ziel, Unregelmäßigkeiten, Meßfehler oder uninteressante kleinräumige Fluktuationen wenn immer möglich zu eliminieren. Hautpsächlich angewendet wird heute eine objektive Methode, welche mit mathematischen Verfahren darauf abzielt, das Integral der Abweichungen des Initialfeldes vom gewünschten korrigierten Feld zu minimieren, wobei das korrigierte Feld eine zusätzliche Bedingung erfüllen muß, wie im vorliegenden Fall die Divergenzfreiheit.

Nach dem Prinzip der Variationsrechnung und einer Methode von Lagrange (siehe SHERMAN 1978) wird nun folgende Gleichung gebildet:

$\mathrm{I}=\int_{\mathrm{v}}\left[\alpha_{1}{ }^{2}\left(\mathrm{u}-\mathrm{u}^{0}\right)^{2}+\alpha_{1}{ }^{2}\left(\mathrm{v}-\mathrm{v}^{0}\right)^{2}+\alpha_{2}{ }^{2}\left(\mathrm{w}-\mathrm{w}^{0}\right)^{2}\right.$

$\left.+\lambda\left(\frac{\delta \mathrm{u}}{\delta \mathrm{x}}+\frac{\delta \mathrm{v}}{\delta \mathrm{y}}+\frac{\delta \mathrm{w}}{\delta \mathrm{z}}\right)\right] \mathrm{dxdydz}$

wobei $\mathrm{u}^{\circ}, \mathrm{v}^{\circ}$ und $\mathrm{w}^{\circ}$ die Windkomponenten des ("gemessenen») Initialwindfeldes, u, v und w diejenigen des justierten Feldes bezeichnen. Mit den Gewichtungsfaktoren $\alpha_{\mathrm{i}}$ können mögliche Unterschiede in den Messgenauigkeiten der einzelnen Faktoren (falls solche bekannt oder zu erwarten sind) berücksichtigt werden.

Berechnet wird also die Summe der gewichteten quadratischen Abweichungen der korrigierten Werte von den interpolierten Werten plus die gesamte Divergenz

$\frac{\delta u}{\delta x}+\frac{\delta v}{\delta y}+\frac{\delta w}{\delta z}$

multipliziert mit dem Lagrange-Multiplikator $\lambda$. Diese Summe wird über den ganzen Modellbereich intergriert. Dabei sollen die Lagrange-Multiplikatoren für jeden Punkt so gewählt werden, daß dieses Integral möglichst klein wird.

Aus den dazugehörenden Euler-Lagrange-Gleichungen, deren Lösungen die Gleichung (1) minimieren (SHERMAN 1978), und den Randbedingungen (mit $\delta_{x}(u)$ als Variation von $\mathrm{u}$ in $\mathrm{x}$-Richtung)

$\lambda \cdot \delta_{\mathrm{x}}(\mathrm{u})=0, \quad \lambda \cdot \delta_{\mathrm{y}}(\mathrm{v})=0, \quad \lambda \cdot \delta_{\mathrm{z}}(\mathrm{w})=0$

an den Grenzflächen senkrecht zur x-, y-bzw. z-Richtung erhält man folgende Differentialgleichung für $\lambda$ :

$\frac{\delta^{2} \lambda}{\delta x^{2}}+\frac{\delta^{2} \lambda}{\delta y^{2}}+\alpha^{2} \cdot \frac{\delta^{2} \lambda}{\delta z^{2}}=-\left({\frac{\delta u^{\circ}}{\delta x}}^{0}+\frac{\delta v^{\circ}}{\delta y}+\frac{\delta w^{\circ}}{\delta z}\right)$.
Gleichung (4) wird für $\lambda$ unter den Randbedingungen (3) gelöst, und das justierte Windfeld wird anschließend mit Hilfe der bestimmten Lagrange-Multiplikatoren für jeden Punkt berechnet (siehe SHERMAN 1978).

Für die Computer-Berechnungen werden die Gleichungen (3) und (4) in eine Finite-Differenzen-Form gebracht:

Der Ausdruck $\delta^{2} \lambda / \delta x^{2}$ wird ersetzt durch

$\left(\lambda_{i+1 \mathrm{jk}}-2 \lambda_{\mathrm{ijk}}+\lambda_{\mathrm{i}-1 \mathrm{jk}}\right) /(\Delta \mathrm{x})^{2}$

der Ausdruck $\delta u^{\circ} / \delta x$ durch

$\left(\mathrm{u}_{\mathrm{i}+\mathrm{j} \mathrm{jk}}^{\mathrm{o}}-\mathrm{u}_{\mathrm{i}-\mathrm{j} \mathrm{jk}}^{\mathrm{o}}\right) /(2 \Delta \mathrm{x})$,

nach SHERMAN (1978). Der Ausdruck $\delta w^{\circ} / \delta z$ ist 0 , da alle Werte für $w^{\circ}$ gleich 0 sind (siehe oben). $\mathrm{i}, \mathrm{j}$ und $\mathrm{k}$ sind ganzzahlig und bezeichnen die Gitterkoordinaten in x-, $y$ - und $z$-Richtung.

Weil $\Delta \mathrm{x}=\Delta \mathrm{y}$ und somit nur das Verhältnis $\Delta \mathrm{z} / \Delta \mathrm{x}$ für die Lösung der Gleichung eine Rolle spielt, wurde $\Delta x=\Delta y=$ 1 und $\Delta z / \Delta x=$ q gesetzt. q bezeichnet somit das Verhältnis des Gitterpunktabstandes in vertikaler zu demjenigen in horizontaler Richtung.

Gleichung (4) erhält demnach folgende Form:

$\lambda_{i+1 \mathrm{jk}}+\lambda_{\mathrm{i}-\mathrm{ljk}}+\lambda_{\mathrm{ij}+\mathrm{lk}}+\lambda_{\mathrm{ij}-\mathrm{lk}}+\left[\left(\alpha^{2} / \mathrm{q}^{2}\right) \cdot\right.$

$\left.\left(\lambda_{i j k+1}+\lambda_{i j k-1}\right)\right]-\left[\left(4+\left(2 \alpha^{2} / q^{2}\right)\right) \cdot \lambda_{i j k}\right]=-1 / 2 \cdot$

$\left(\mathrm{u}_{\mathrm{i}+1 \mathrm{jk}}^{\mathrm{o}}-\mathrm{u}_{\mathrm{i}-\mathrm{ljk}}^{\mathrm{o}}+\mathrm{v}_{\mathrm{ij}+\mathrm{lk}}^{\mathrm{o}}-\mathrm{v}_{\mathrm{ij}-\mathrm{lk}}^{\mathrm{o}}\right)$

Die Randbedingungen werden wie folgt integriert:

Für Durchflußgrenzen am Rand des Berechnungsbereiches wird $\lambda=0$ gesetzt. Taucht ein Punkt der Topographie in Gleichung (7) auf, so wird die Bedingung für eine "geschlossene» Grenze, $\delta \lambda / \delta n=0$, angewendet. Ist zum Beispiel im Differenzschema für den Punkt (i,j, k) der Punkt $(i+1, j, k)$ ein Topographiepunkt, so wird die erwähnte Bedingung nach SHERMAN (1978) durch ein Drei-PunktDifferenzschema ausgedrückt:

$\delta \lambda / \delta x\left(3 \lambda_{i+1 j \mathrm{k}}-4 \lambda_{i \mathrm{jk}}+\lambda_{\mathrm{i}-\mathrm{j} \mathrm{jk}}\right) / 2 \Delta \mathrm{x}=0$,

und entsprechend in Gleichung (7) eingesetzt. Auf diese Weise wird für jeden «inneren» Gitterpunkt (i, j, k), welcher über der Topographie und nicht an einer Bereichsgrenze liegt, die Gleichung (7) aufgestellt, wobei die Werte für $\lambda$ an den Grenzen wie oben beschrieben ersetzt werden.

Es entsteht also ein Gleichungssystem für die $\lambda$-Werte für alle «inneren» Punkte. Dieses wird mit Hilfe von Stones "Strongly Implicit Procedure» durch das Programm D03ECF der NAG-Programmbibliothek (NAG, 1987) interativ gelöst und berechnet für jeden «inneren» Punkt den Wert $\lambda$.

Das justierte Windfeld wird nachfolgend berechnet aus

$\mathrm{u}_{\mathrm{ijk}}=1 / 4\left(\mathrm{u}_{\mathrm{i}+1 \mathrm{jk}}^{\mathrm{o}}+2 \mathrm{u}^{\mathrm{o}}{ }_{\mathrm{ijk}}+\mathrm{u}^{\mathrm{o}}{ }_{\mathrm{i}-\mathrm{j} \mathrm{jk}}\right)$

$+\left[\left(\lambda_{i+1 j k}-\lambda_{i-1 \mathrm{ik}}\right) / 2 \Delta \mathrm{x}\right]$

$\mathrm{v}_{\mathrm{ijk}}=1 / 4\left(\mathrm{v}_{\mathrm{ij}-\mathrm{lk}}^{\mathrm{o}}+2 \mathrm{v}_{\mathrm{ijk}}^{\mathrm{o}}+\mathrm{v}_{\mathrm{ij}-1 \mathrm{k}}^{\mathrm{o}}\right)$

$+\left[\left(\lambda_{i j-1 k} \lambda_{i j-1 k}\right) / 2 \Delta y\right]$ 
$\mathrm{w}_{\mathrm{ijk}}=\alpha^{2} \cdot\left[\left(\lambda_{\mathrm{i}+\mathrm{j} \mathrm{jk}}-\lambda_{\mathrm{i}-\mathrm{j} \mathrm{jk}}\right) / 2 \Delta \mathrm{z}\right]$

(Eine Differential-Formulierung von oben nicht im einzelnen aufgeführten Lagrange-Gleichungen).

Gleichung (11) für die Vertikalgeschwindigkeit ist deshalb vereinfacht, weil keine Initialwerte vorhanden sind.

\subsection{Trajektorien}

Um die Trajektorien zu berechnen, wird vom gewünschten Starttermin an jede Stunde ein Windfeld gemäß obenstehender Beschreibung berechnet. An den interessanten Punkten, d. h. dort, wo sich das untersuchte Partikel gerade befindet, werden die Geschwindigkeitskomponenten räumlich linear interpoliert. Für den Raum zwischen der untersten Modellschicht und der Topographie wird mit einem «Power-Law»-Profil (siehe oben) gerechnet. Hier liegt eines der größten Probleme des Trajektorienmodells: Nämlich zu verhindern, daß die Trajektorie im Boden verschwindet. Da das Windfeld in Bodennähe häufig sehr kompliziert ist und vom Modell nicht repräsentiert werden kann, muß mit Kunstgriffen versucht werden, dies zu unterbinden. Damit wird jedoch die Aussagekraft einer solchen Trajektorie sehr fraglich. Mit einem so grobmaschigen Modell lassen sich Trajektorien in Bodennähe (ca. unterste $100 \mathrm{~m}$ ) kaum auf vernünftige Art und Weise berechnen.

Der Zeitschritt ist variabel wählbar. Es hat sich gezeigt, daß kleinere Zeitschritte bis zu einer gewissen Grenze (ca. 10 Min.) Verbesserungen mit sich bringen, daß aber das Resultat bei einer weiteren Verkleinerung normalerweise nicht mehr stark beeinflußt wird. Eine Ausnahme bilden hier Trajektorien in der Nähe der Topographie: Bei hohen Windgeschwindigkeiten kann ein Partikel die von der Topographie beeinflußten (wenigen) Schichten in einem Schritt "durchfliegen» und somit natürlich in den Hügel "rasen», hier ist oft eine starke Verkleinerung des Zeitschrittes nötig, um diesem Problem zu begegnen. Für die Berechnung werden als erstes die Geschwindigkeitskomponenten $\mathrm{u}_{\mathrm{o}}, \mathrm{v}_{\mathrm{o}}, \mathrm{w}_{\mathrm{o}}$ zum Zeitpunkt und am Ort des Startes berechnet. Dann wird eine erste Näherung des Ortes nach einem Zeitschritt bestimmt nach der Formel $\quad \mathrm{P}_{1}\left(\mathrm{x}_{1}, \mathrm{y}_{1}, \mathrm{z}_{1}\right)=\left(\mathrm{x}_{\mathrm{o}}+\mathrm{u}_{\mathrm{o}} \Delta \mathrm{t}, \mathrm{y}_{\mathrm{o}}+\mathrm{v}_{\mathrm{o}} \Delta \mathrm{t}, \mathrm{z}_{\mathrm{o}}+\mathrm{w}_{\mathrm{o}} \Delta \mathrm{t}\right)$.

Danach werden die Geschwindigkeitskomponenten $u_{1}$, $v_{1}, w_{1}$ im Punkt $P_{1}$ zur Zeit $t+\Delta$ t berechnet, und die zweite Approximation $\mathrm{P}_{2}$ des fraglichen Ortes ist gegeben durch

$\mathrm{P}_{2}\left(\mathrm{x}_{2}, \mathrm{y}_{2}, \mathrm{z}_{2}\right)=\left(\mathrm{x}_{\mathrm{o}}+\mathrm{u}_{\mathrm{o}} \Delta \mathrm{t} / 2+\mathrm{u}_{1} \Delta \mathrm{t} / 2, \mathrm{y}_{\mathrm{o}}+\mathrm{v}_{\mathrm{o}} \Delta \mathrm{t} / 2+\mathrm{v}_{1} \Delta \mathrm{t} / 2, \mathrm{z}_{\mathrm{o}}\right.$

$\left.+\mathrm{w}_{0} \Delta \mathrm{t} / 2+\mathrm{w}_{1} \Delta \mathrm{t} / 2\right)$

Dieser Schritt wird in der folgenden Art iterativ wiederholt, so daß

$P_{n}\left(x_{n}, y_{n}, z_{n}\right)=\left(x_{o}+u_{0} \Delta t / 2+u_{n-1} \Delta t / 2, y_{0}+v_{o} \Delta t / 2\right.$

$\left.+\mathrm{v}_{\mathrm{n}-1} \Delta \mathrm{t} / 2, \mathrm{z}_{\mathrm{o}}+\mathrm{w}_{\mathrm{o}} \Delta \mathrm{t} / 2+\mathrm{w}_{\mathrm{n}-1} \Delta \mathrm{t} / 2\right)$

bis der Wert $\left(x_{n}-x_{n-1}\right)^{2}+\left(y_{n}-y_{n-1}\right)^{2}+\left(z_{n}-z_{n-1}\right)^{2}$, das heißt das Quadrat der Distanz zwischen Punkt $P_{n}$ und $P_{n-1}$, kleiner ist als ein festgelegtes Konvergenzkriterium. Diese Methode wurde von PETTERSEN (1956) vorgeschlagen.
Für die Bestimmung von Rückwärtstrajektorien wird mit der gleichen Methode, jedoch mit den negativen Geschwindigkeitskomponenten (z. B. $-u_{o},-v_{0},-w_{o}$ statt $u_{o}$, $v_{o}, w_{o}$ ) gerechnet.

\section{Modelltest}

Das Modell wurde auf verschiedene Arten getestet: Als erstes wurde eine Modelltopographie mit künstlichen, regelmäßigen topographischen Elementen in ein in allen Dimensionen homogenes Windfeld gesetzt, um den Einfluß der mathematischen «Justierung» zu testen. Es hat sich dabei gezeigt, daß das Umströmen der Hügel gut in das Modell eingebaut wird, daß das Überströmen ebenfalls bewältigt wird, mit jedoch zu geringen Vertikalgeschwindigkeiten. Von der Topographie «beeinflußt» werden jedoch nur die zwei bis drei dem Element am nächsten liegenden Schichten (horizontal wie vertikal). Beide Probleme, sowohl die zu kleinen Vertikalgeschwindigkeiten als auch die zu wenig weit reichenden Einflüsse der Topographie, hängen sehr wahrscheinlich mit der diskretisierten Auflösung (Einzelpunkte statt kontinuierliche Werte) zusammen, d. h. mit nicht in die Berechnung einbezogenen subskaligen Phänomenen sowie mit den nicht berechenbaren Vorgängen im Bereich zwischen der untersten Modellschicht und der Topographie. Andererseits werden gewisse in der Natur beobachtbare Phänomene wie Lee-Wellen rein aufgrund der Bedingung der Massenkonsistenz erzeugt.

Um die Modellresultate zu testen, wurden die für die Standorte einzelner geeigneter Klimastationen errechneten Werte mit den von diesen Stationen gemessenen verglichen. Leider sind nur sehr wenige Klimastationen für einen solchen Vergleich geeignet, da sich die Windmesser abgesehen von der Frage der Repräsentativität häufig nur wenig über Dachhöhe ( 1 bis $3 \mathrm{~m}$ ) befinden und deshalb durch Turbulenzeffekte rund um das Gebäude beeinflußt werden. Von den vier verbliebenen Stationen (Unterbözberg, Menzberg, Oeschberg, Haidenhaus) wurden im Mittel Abweichungen der Modellresultate von 14 bis 39 Grad für die Windrichtung und von 30 bis $56 \%$ für die Windgeschwindigkeiten beobachtet. Diese Werte sind angesichts der für die Vergleiche verwendeten Situationen mit relativ tiefen Windgeschwindigkeiten ( 1,5 bis $3 \mathrm{~m} / \mathrm{s}$ ) und der mit Interpolationen verbundenen Unsicherheiten als recht gut zu beurteilen.

\section{Modellresultate}

\subsection{Windfelder}

Um die Möglichkeiten und Grenzen des Modells zu zeigen, sind im folgenden ein Ausschnitt des Windfeldes über der Region Bern-Thun und verschiedene Trajektorien beschrieben. Es handelt sich um Windfelder vom 27. Dezember zwischen 9 und 21 Uhr, also um Trajektorien über 12 Stunden. Innerhalb dieser zwölf Stunden 


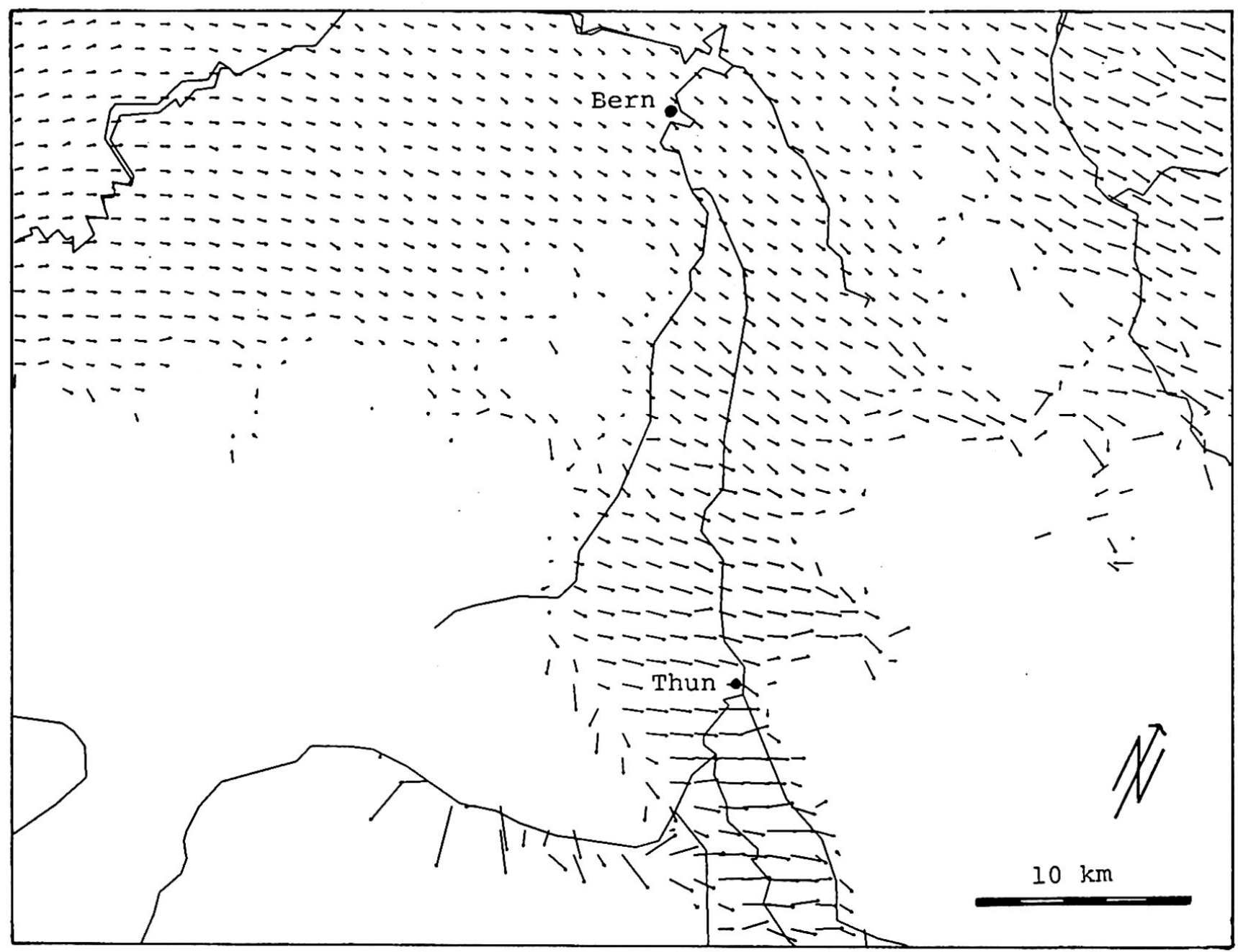

Fig. 3 WITRA-Windfeld vom 27.12.88, 9 Uhr MEZ auf $900 \mathrm{~m}$ ü. M.

wechseln die Winde laufend Geschwindigkeit und Richtung. Als Beispiele sind die Windfelder von 9 Uhr auf $900 \mathrm{~m}$ ü. M. (Fig. 3) und von $15 \mathrm{Uhr}$ auf $1000 \mathrm{~m}$ ü. M. (Fig. 4) dargestellt. Auf diesen beiden Figuren sind die unterschiedlichen Windverhältnisse deutlich zu erkennen. Im Laufe des Tages drehen die Winde von West auf Ost mit gegen Abend steigenden Geschwindigkeiten.

Zu den Windfeldern im Detail: Aufbeiden Figuren ist relativ gut zu erkennen, was ein solches Windfeld bieten kann und was nicht. Relativ gut simuliert wird die Umströmung von Hügeln im Alpenvorland (z. B. Längenberg südwestl. von Bern). Problematisch wird das angewandte Verfahren in tiefen, engen Tälern, deren Breite nur noch durch ein bis zwei Gitterpunkte repräsentiert wird (z. B. das Simmental). Die vielen «Ecken» (bedingt durch die Darstellungsart des Terrains) führen in gewissen Fällen zu recht chaotischen Windfeldern. Allerdings ist hier zu berücksichtigen, daß in Wirklichkeit in solchem Terrain sehr starke Verwirbelungen und Gegenströmungen entstehen können, die sich jedoch in einer
Größenordnung unterhalb des Gitterpunktabstandes bewegen. Solche Windsysteme sind mit dem Modell sowieso nicht mehr erfaßbar. Die Berechnung von Trajektorien ist in solchen Gebieten nicht mehr zweckmäßig.

\subsection{Trajektorien}

In Fig. 5 sind drei Trajektorien dargestellt, welche zum gleichen Zeitpunkt ( $9 \mathrm{Uhr}$ ) und am selben Ort ( 2 km südöstlich von Münsingen), aber in verschiedenen Höhen $(800,900$ und $1000 \mathrm{~m}$ ü. M.) gestartet worden sind. Die Flugdauer beträgt für alle Flugbahnen 12 Std. Die unterschiedlichen Winde in verschiedenen Höhen (vor allem bezüglich Geschwindigkeit) führen zu sehr unterschiedlichen Flugbahnen. Große Unterschiede sind vor allem in der Transportdistanz zu erkennen, während alle Trajektorien eine ähnliche Form aufweisen: Zuerst erfolgt eine Teilchenverschiebung nach Osten und dann in einem gegen Süden gerichteten Bogen nach Westen. Auffallend ist, daß die Trajektorien auf 800 und $900 \mathrm{~m}$ durch das Tal bei Riggisberg führen (siehe Fig. 3), während die Bahn 


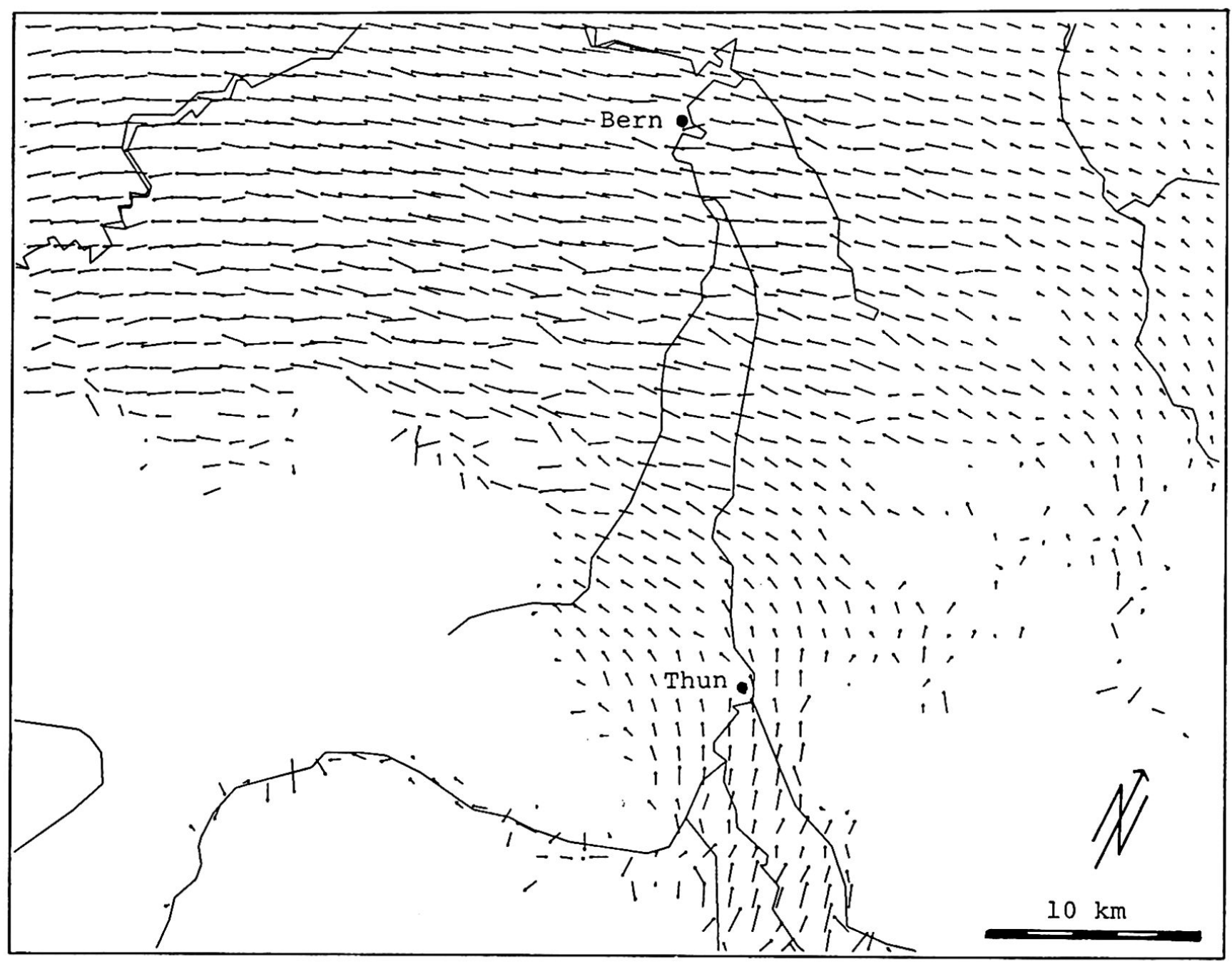

Fig. 4 WITRA-Windfeld vom 27.12.88, 15 Uhr MEZ auf 1000 m ü. M.

auf $1000 \mathrm{~m}$ ü. M. über den nördlich liegenden Hügel führt, was für die gute Simulation der Umströmung von Terrainerhöhungen spricht. Hier zeigt sich, daß mit dem vorliegenden Modell die Berechnung von Trajektorien gegenüber der bloßen Verwendung von Bodenmeßstationen und Profilmessungen doch erheblich verbessert wird. Der Vergleich mit gemessenen Trajektorien, d. h. mit Ballon-Flugbahnen, steht z. Z. noch aus. Auch bei der Berechnung der Trajektorien hat sich wieder gezeigt, daß tiefe Windgeschwindigkeiten große Probleme bieten: Werden am Zielpunkt von berechneten Flugbahnen zum Zeitpunkt der Ankunft Rückwärtstrajektorien über dieselbe Zeitdauer gestartet, so müßte im Idealfall der Ausgangspunkt der Trajektorie erreicht werden. Dies ist jedoch nur bei Windgeschwindigkeiten über 1,5 bis $2 \mathrm{~m} / \mathrm{s}$ mehr oder weniger der Fall (Abweichungen von unter $1 \mathrm{~km}$ auf Flugdistanzen von 60 bis $70 \mathrm{~km}$ ). Bei kleinen Windgeschwindigkeiten treten viel größere Abweichungen auf, welche allein durch das Rechenverfahren bedingt sind.

\section{Anwendungsmöglichkeiten des Modells}

Wie bereits in der Einleitung erwähnt, ist die Anwendbarkeit des Modells auf gewisse meteorologische Situationen beschränkt. Wegen der begrenzten räumlichen Auflösung einerseits der Meßresultate und andererseits des Modells können nur Windfelder beschrieben werden, die im Skalenbereich des Gitterpunktabstandes einigermaßen homogen sind. Diese Bedingung ist nur bei genügend hohen Windgeschwindigkeiten (größer als 1,5 bis $2 \mathrm{~m} / \mathrm{s}$ ) erfüllt. Bei kleineren Geschwindigkeiten ist bereits die Extrapolation der Meßwerte von $10 \mathrm{~m}$ über Boden an die Obergrenze der «Surface Layer» ( 50 bis $100 \mathrm{~m}$ über Boden) ein Problem. Ebenfalls nicht erfaßt werden kleinräumige Luftbewegungen und Turbulenzen in der Nähe der Topographie (auch bei starkem Wind). Diese Tatsache wirkt sich vor allem bei der Berechnung von Trajektorien aus, wenn sich das zur Diskussion stehende Partikel in der Nähe der Topographie bewegt. In einem solchen Fall wird die Berechnung einer auch nur annä- 


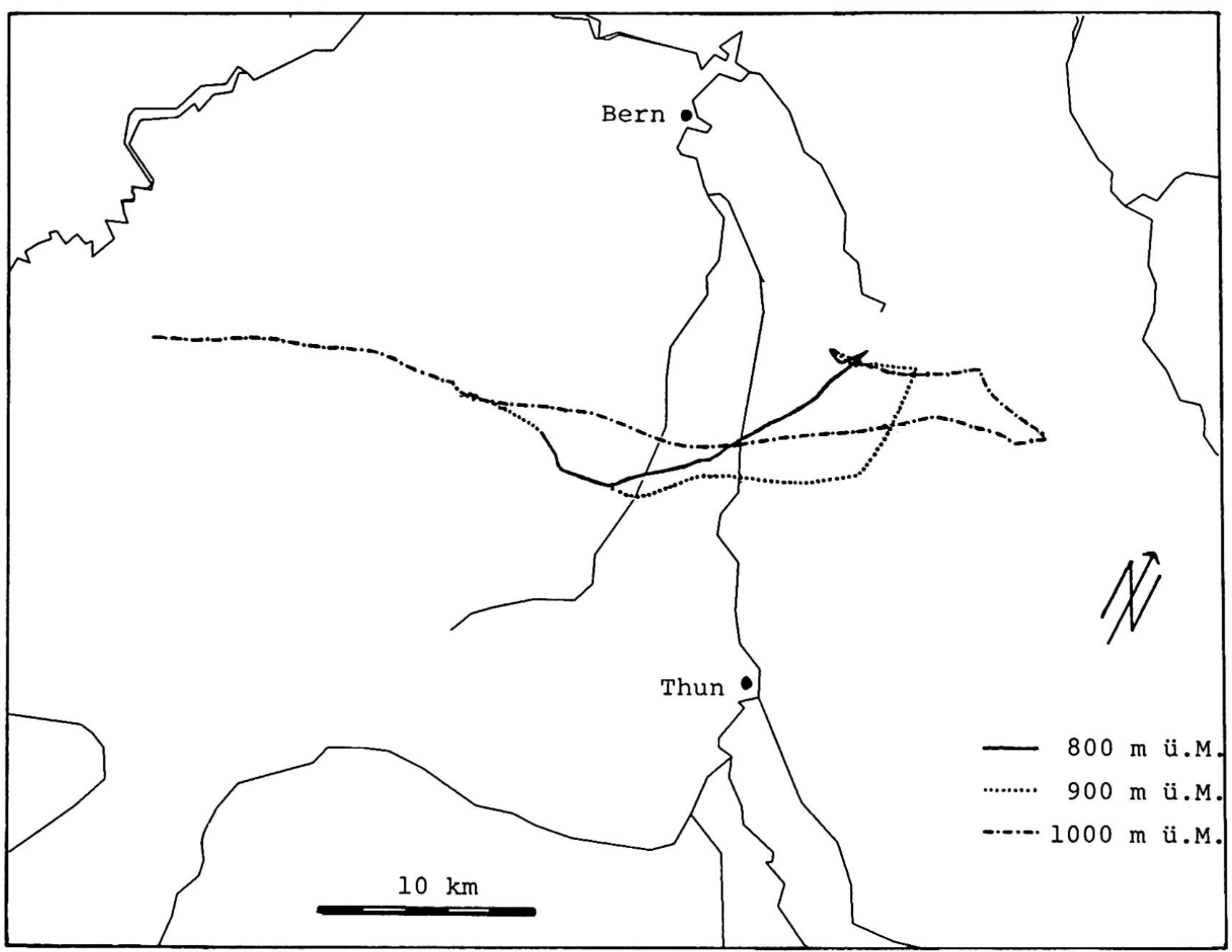

Fig. 5 Trajektorien vom 27.12.88, Startpunkt 9 Uhr MEZ, Koordinaten 610000/190000 (südöstlich von Münsingen BE) auf 800,900 und $1000 \mathrm{~m}$ ü. M. über jeweils 12 Stunden

hernd der Wirklichkeit entsprechenden Flugbahn illusorisch. Hier kann mit einigen rechentechnischen Kunstgriffen nur noch verhindert werden, daß das Partikel in der Topographie verschwindet. Die Anwendung des Modells wurde bisher aus den erwähnten Gründen auf winterliche Hochnebellagen mit mehr oder weniger neutraler Temperaturschichtung beschränkt. Eine mögliche Anwendung für sommerliche Hochdrucklagen bei hohen Windgeschwindigkeiten (z. B. Bisen-Situationen) oder allenfalls auch für Westwindfälle muß noch geprüft werden.

\section{Literatur}

NAG (1987): NAG FORTRAN Mini Manual, Mark 12. The Numerical Algorithms Group Limited, Oxford.
NEU, U. (1990): WITRA - Ein Verfahren zur Erzeugung von dreidimensionalen, divergenzfreien Windfeldern über der komplexen Topographie des schweizerischen Mittellandes aus Routine-Meßdaten. Geogr. Inst. Univ. Bern.

PETTERSSEN, S. (1956): Weather analysis and forecasting, 2nd ed., vol. I. McGraw-Hill, New York.

RICKLI, B., FILLIGER, P., TROXLER, F.X., PFEIFER, R., BRUNNER, T., ESTERMANN, A. und SALVISBERG E. (1989): Das Ausbreitungsklima der Innerschweiz - Studie über die Durchlüftungsverhältnisse in den Kantonen Luzern, Nidwalden, Obwalden, Schwyz, Uri und Zug. Geogr. Inst. Univ. Bern, 2 Bde.

SASAKI, Y. (1958): An objective analysis based on the variational method. J. Meteor. Soc. Japan, 36, 77-88.

SHERMAN, C. A. (1978): A mass-consistent model for wind fields over complex terrain. J. Appl. Meteor., 17, 312-319. 